\title{
Penetrating Eyelid and Ocular Fishhook-Related Injury
}

\author{
Janejit Choovuthayakorn Pimploy Chavengsaksongkram \\ Nawat Watanachai Winai Chaidaroon \\ Chiang Mai University Hospital, Chiang Mai, Thailand
}

\section{Keywords}

Ocular fishhook injury $\cdot$ Sport-related eye injury $\cdot$ Fishhook eye trauma

\begin{abstract}
Purpose: To report removal techniques and outcomes for a patient with penetrating eyelid and cornea complicating localized retinal detachment from a fishhook. Methods: This is a case report of a 35-year-old man who presented with a fishhook embedded in his right upper eyelid and globe while participating in a fishing competition. On initial examination, his right eyelid was opened with difficulty and limited evaluation could be performed. Results: In an operating room, a shank was transected and removed from a lacerated eyelid by a back-out technique. Then, a barbed hook was noticed to penetrate through temporal peripheral cornea, iris, and entrapped within ciliary body behind the lens. A successful surgical removal of a fishhook was performed within a primary operation. After that, additional operations to repair injured ocular tissues including a localized retinal detachment were performed. The patient achieved a bestcorrected visual acuity of 20/20 at 10-month follow-up. Conclusions: The visual prognosis for a fishhook-related ocular injury is associated with various variables including size and location of lacerated wound and technique to remove a fishhook. In this case, a patient could achieve a favorable final visual and anatomical outcome. This removal technique could be taken into consideration when encountering a fishhook-related eye injury patient.
\end{abstract}

(C) 2019 The Author(s)

Published by S. Karger AG, Basel 


\section{Case Reports in Ophthalmology}

\section{Introduction}

Ocular trauma is the leading global cause of blindness and visual morbidity in the working-age population [1]. Several causes of ocular trauma have been reported, in which sportrelated injury was one. In 2005, the two most commonly reported sporting-related eye injuries on the United States Eye Injury Register (USEIR) were those related to baseball (22\%) followed by fishing (20\%) [2]. Despite the prevalence of eye trauma related to a fishhook etiology, there is limited published information regarding the penetrating fishhook eyelid and globe injuries. Visual morbidity following a fishhook eye trauma has been reported as a result of corneal scarring, retinal detachment, and endophthalmitis; however, the prompt and appropriate surgical managements could prevent these devastating consequences [2, 3]. This study reports on one interesting case of simultaneous eyelid and ocular injuries caused by a double prong fishing hook in a bystander demonstrating clinical presentations, surgical removal techniques, and subsequent outcomes.

\section{Case Report}

A 35-year-old man was referred to the hospital with an eye injury in which one prong of a double prong fishhook had stabbed through the upper eyelid and cornea and was embedded in the temporal area of the right eyeball (Fig. 1a). The accident occurred while he was a bystander observing a fishing competition. No attempt was made to remove it. On initial presentation, the patient reported visual acuity of hand movement in the injured eye. Detail evaluation of anterior and posterior segments could not be properly performed as the upper eyelid was stuck with the fishhook. Intravenous ceftazidime dosing $1 \mathrm{~g}$ every $8 \mathrm{~h}$ and vancomycin dosing $1 \mathrm{~g}$ every $12 \mathrm{~h}$ were given to the patient.

Under general anesthesia, the hook shank was firmly grasped and a wire cutter was inserted beneath the upper lid, above the cornea, to cut the bend of the fishhook (Fig. 1b). The proximal part of the fishhook was removed backward through the entry wound of the upper eyelid and it was, then, possible to open the eyelid fully (Fig. 1c). On examination, the hook was seen to have perforated the temporal peripheral cornea and traversed through the iris and lens zonule and was tightly entrapped within the ciliary body inside the eyeball. No exit wound was observed. The point of the hook had adhered approximately $3 \mathrm{~mm}$ posterior to the limbus. Traumatic cataract and hyphema were noted. Conjunctival peritomy was performed at the temporal limbal site, from 7 to 11 o'clock, corresponding to the traumatic conjunctiva. The remaining end of the bent hook was gently pressed outward against the sclera to locate the embedded hook. Incision was made through the tented sclera starting from the point of the fishhook to a limbus using a straight surgical blade No. 15. The entire barbed hook was, then, advanced forward and rotated out through the scleral wound. The iris and vitreous were noticed at the surgical site. The corneal wound was repaired with 10.0 nylon, while the limbal and scleral wounds were sutured with 8.0 nylon. The iris was repositioned and the anterior chamber was reformed using balanced salt solution. The vitreous was managed. The lacerated eyelid was approximately repaired. During the final step, intravitreal injections of vancomycin $(1 \mathrm{mg} / 0.1 \mathrm{~mL})$ and ceftazidime $(2.25 \mathrm{mg} / 0.1 \mathrm{~mL})$ were given. The appearance of the fishhook is shown in Figure 1d. After the operation, $1 \%$ of prednisolone acetate and $0.5 \%$ of levofloxacin eye drops were given topically four times a day and tapered over a period of 4 weeks. In addition, intravenous ceftazidime (1 g every $8 \mathrm{~h}$ ) and vancomycin ( $1 \mathrm{~g}$ every $12 \mathrm{~h}$ ) 


\section{Case Reports in Ophthalmology}

were administered continuously for 5 days. Due to high prevalence of post-traumatic infection in this region, systemic steroid was not prescribed.

On day 1 postoperatively, anterior segment examination revealed secured corneal and scleral wounds, a peak pupillary margin, hyphema, and a traumatic cataract (Fig. 2). The Bscan showed signs of localized retinal detachment without choroidal injury. Then, oral steroid was not prescribed. A week after the primary operation, a combined lensectomy and pars plana vitrectomy were done. A mid-peripheral retinal tear, from a point of the fishhook, and a surrounded localized inferotemporal retinal detachment were repaired. A 5,700-centistoke silicone oil was used as a tamponade at the end of surgery. The removal of the silicone oil with secondary intraocular lens implantation were carried out 6 months after injury. At month 10 , the best corrected visual acuity was 20/20. Temporal peripheral corneal scar was seen. The retina appeared completely attached with inferotemporal retinal scar (Fig. 3). No other significant complication was noted.

\section{Discussion and Conclusions}

Previous literature has reported the cases of fishing-related eye injuries. These reports are, of necessity, variable in terms of surgical procedures depending on type, location, and severity of ocular structure involvements [4-10]. Information regarding the appearances of the fishhook, including barb and shank type, is also vital to inform the surgical plan. Alfaro et al. [2] reported that $44 \%$ of fishhook injury cases were of open globe injury type with the most common presentation being corneal laceration. Aiello et al. [3] found that anterior segment damage was most common in penetrating fishhook injuries with eyelid involvement by separated hook noted in 1 of 5 cases.

Techniques for removal of an embedded fishhook from ocular tissue have been published. For a penetrating cornea with no posterior segment injury, surgical removal methods including advance, advance-and-cut, and back-out techniques have been proposed [8-10]. Furthermore, a modified back-out technique, in which a primary scleral wound was enlarged and a hook was removed backwards through the entry wound, was shown in medical reports [1113]. Recently, Chao et al. [14] demonstrated a cut-and-push-through technique for management of penetrating corneal fishhook injury. In this case, the fishhook perforated through the peripheral cornea and was lodged in the ciliary body. Performing a controlled surgical exit wound was used to minimize additional trauma of engaged ocular tissues during removal procedures. Favorable results were achieved at a final visit. In conclusion, the prognosis of fishhook-related ocular injury varies in size, location, and method of removal of the foreign body. We report a removal technique which enabled the successful visual and anatomical outcomes. This technique may be taken into consideration when encountering a fishhook-related ocular injury patient. However, to minimize the occurrence of fishhook-related eye trauma, a careful eye protection for fishing participants and bystanders should be advised. 


\section{Case Reports in Ophthalmology}

Choovuthayakorn et al.: Penetrating Eyelid and Ocular Fishhook-Related Injury

\section{Statement of Ethics}

The authors have no ethical conflicts to declare.

\section{Disclosure Statement}

The authors declare that they have no conflict of interest.

\section{References}

1 Negrel AD, Thylefors B. The global impact of eye injuries. Ophthalmic Epidemiol. 1998 Sep;5(3):143-69.

2 Alfaro DV 3rd, Jablon EP, Rodriguez Fontal M, Villalba SJ, Morris RE, Grossman M, et al. Fishing-related ocular trauma. Am J Ophthalmol. 2005 Mar;139(3):488-92.

3 Aiello LP, Iwamoto M, Guyer DR. Penetrating ocular fish-hook injuries. Surgical management and long-term visual outcome. Ophthalmology. 1992 Jun;99(6):862-6.

4 Grand MG, Lobes LA Jr. Technique for removing a fishhook from the posterior segment of the eye. Arch Ophthalmol. 1980 Jan;98(1):152-3.

5 Mandelcorn MS, Crichton A. Fish hook removal from vitreous and retina. Case report. Arch Ophthalmol. 1989 Apr;107(4):493.

6 Levy J, Lifshitz T. Eyelid fish-hook injury. Int Ophthalmol. 2001;24(5):297-8.

7 Moore DB, Slabaugh MA. Fishing-related ocular trauma in the Pacific Northwest: hook, line and sinker. Int Ophthalmol. 2012 Dec;32(6):589-93.

8 Yildirim N, Kabadere E, Ermis Z. Perforating corneal injury with a fish hook. Ophthalmic Surg Lasers Imaging. 2008 Mar-Apr;39(2):137-9.

9 Agrawal R, Laude A, Taneja M. Fish-hook injury of the eye. Int Ophthalmol. 2012 Jun;32(3):269-71.

10 Yüksel N, Elibol O, Cağlar Y. Penetrating corneal fish-hook injury. Ophthalmologica. 1994;208(2):112-3.

11 Iannetti L, Tortorella P. Penetrating fish-hook ocular injury: management of an unusual intraocular foreign body. Case Rep Med. 2014;2014:901285.

12 Ding X, Liu Z, Lin Y, Yang Y. Perforating ocular fishhook trauma: a case report. Clin Exp Optom. 2018 Mar;101(2):297-8.

13 Knox FA, Chan WC, McAvoy CE, Johnston SE, Bryars JH. Penetrating ocular injuries from fish-hooks. Int Ophthalmol. 2004 Oct-Dec;25(5-6):291-4.

14 Chao DL, Erickson BP, Rachitskaya AV. Penetrating ocular fishhook injury. JAMA Ophthalmol. 2015 Mar;133(3):347-8. 


\section{Case Reports in Ophthalmology}

\begin{tabular}{l|l}
\hline Case Rep Ophthalmol 2019;10:41-46 \\
\hline DOI: 10.1159/000496382 & $\begin{array}{l}\text { (c) 2019 The Author(s). Published by S. Karger AG, Basel } \\
\text { www.karger.com/cop }\end{array}$ \\
\hline
\end{tabular}

Choovuthayakorn et al.: Penetrating Eyelid and Ocular Fishhook-Related Injury


Fig. 1. Color photograph showing an injured eye at initial presentation (a), during surgical removal of a shank hook in the operating room (b), and after complete removal of the fishhook (c). $\mathbf{d}$ Appearance of the double prong fishhook causing the injury.

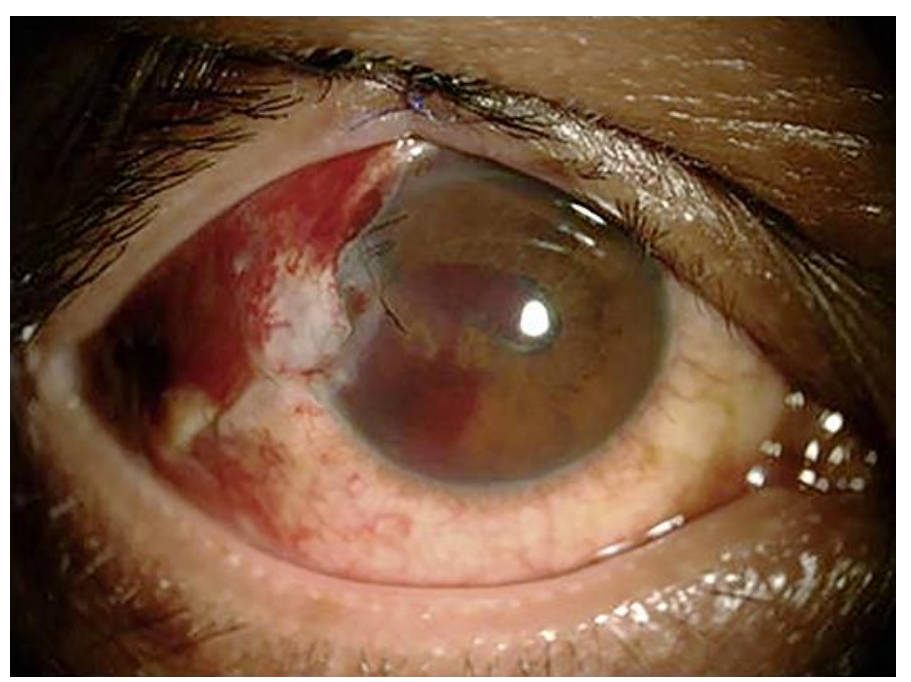

Fig. 2. Color photograph showing anterior segment findings following the primary operation. 
Case Reports in
Ophthalmology

Case Rep Ophthalmol 2019;10:41-46

DOI: $10.1159 / 000496382$

(c) 2019 The Author(s). Published by S. Karger AG, Basel www.karger.com/cop

Choovuthayakorn et al.: Penetrating Eyelid and Ocular Fishhook-Related Injury
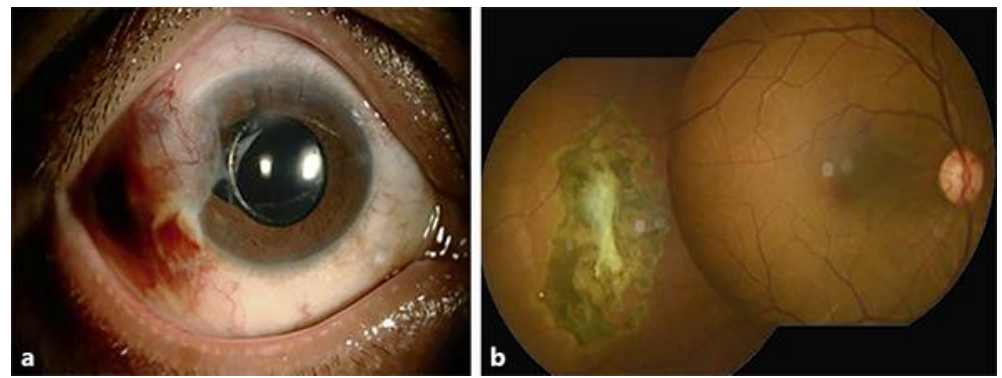

Fig. 3. Color photograph at 10-month follow-up showing anterior segment (a) and fundus finding (b). 